\title{
Protective effect of gastrodin on myocardial ischemia-reperfusion injury and the expression of Bax and Bcl-2
}

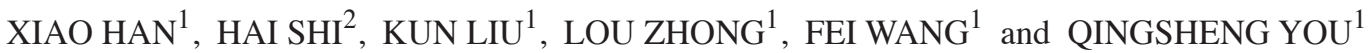 \\ ${ }^{1}$ Department of Cardiothoracic Surgery, Affiliated Hospital of Nantong University; ${ }^{2}$ Department of \\ Cardiothoracic Surgery, The Third People's Hospital of Nantong, Nantong, Jiangsu 226001, P.R. China
}

Received November 14, 2018; Accepted March 4, 2019

DOI: $10.3892 /$ etm.2019.7512

\begin{abstract}
The protective effects of gastrodin on myocardial ischemia-reperfusion injury in rats and the underlying mechanism were investigated. Sprague Dawley (SD) rats were randomly divided into three groups, of which the gastrodin group was treated with gastrodin, and the other two groups were treated with normal saline. In the myocardial ischemiareperfusion model group, myocardial ischemia was induced by ligation of the left anterior descending coronary artery, and myocardial reperfusion was performed by ligature removal. Only thread without ligation for the sham operation group was conducted. The rats were euthanized 8 days after surgery. Heart tissues were harvested and used for measurement of apoptotic rate and expression levels of apoptosis-related proteins. Serum levels of cytokines were measured also using blood samples. Compared with the myocardial ischemia-reperfusion model group, significant reduction of cardiomyocyte apoptosis was observed in the gastrodin group $(\mathrm{P}<0.05)$. In the gastrodin group, the protein and mRNA expression levels for Bax and activated caspase-3 decreased, while for Bcl-2 increased $(\mathrm{P}<0.05)$. Gastrodin can downregulate inflammatory cytokines $(\mathrm{P}<0.05)$ and upregulate anti-inflammatory cytokines such as IL-10 $(\mathrm{P}<0.05)$ in serum of SD rats. Therefore, gastrodin played a protective role in myocardial ischemia-reperfusion injury by regulating the expression levels of apoptosis-related signaling proteins and inflammatory cytokines.
\end{abstract}

\section{Introduction}

Myocardial ischemia has a high incidence of morbidity and mortality worldwide (1). It has been shown that acute myocardial ischemia is a leading cause of death (2). In addition to sudden death, myocardial ischemia also leads to various clinical manifestations such as cardiac myocyte hypertrophy (3-5).

Correspondence to: Dr Qingsheng You, Department of Cardiothoracic Surgery, Affiliated Hospital of Nantong University, 20 Xisi Road, Nantong, Jiangsu 226001, P.R. China

E-mail: qbk5bs@163.com

Key words: gastrodin, myocardial ischemia-reperfusion injury, Bax, Bcl-2
Therapeutic interventions in myocardial ischemia have been explored extensively and many treatment options have been published. Myocardial ischemia-reperfusion is an effective treatment method for acute myocardial ischemia, which can reduce myocardial infarction size and relieve symptoms of heart failure (6). However, myocardial ischemia-reperfusion injury exists and remains a complex problem. Dysregulation of many signaling pathways have been suggested to be associated with heart damage caused by myocardial ischemia, especially the dysregulation of intracellular signaling pathways. Signaling disorders leading to cell proliferation, differentiation, autophagy and apoptosis under physiological or pathological conditions may be closely related to cardiac damage (7).

As a traditional Chinese herbal medicine, rhizome gastrodiae has been used in China for thousands of years. According to previous studies, rhizome gastrodiae has nootropic, analgesic and anti-inflammatory effects and can improve microcirculation and general circulatory functions $(8,9)$. Gastrodin is the major active ingredient in rhizome gastrodiae, which demonstrates a high application value (10). It was reported that gastrodin has neuroprotective effects on cultured cortical neurons (11). Gastrodin can inhibit expression of pro-inflammatory cytokines and cyclooxygenase-2 (12). Gastrodin can regulate signaling pathways involved in inflammatory responses. Studies have suggested that the beneficial effects of gastrodin may be derived from its antioxidant properties (13). GAS can protect primary cultured rat hippocampal neurons from beta amyloid-induced neurotoxicity (14). In this study, the protective effect of gastrodin on myocardial ischemia-reperfusion injury and the possible molecular mechanism were explored, aiming to provide a reference for the treatment of this disease.

\section{Materials and methods}

Experimental animals and grouping. Male 7-8-week-old Sprague Dawley (SD) rats, weighing 210-250 g, were raised in a cage, with free access to food and water, on a 12:12-h light/dark cycle for a week. The temperature was $22 \pm 2^{\circ} \mathrm{C}$, and the relative humidity was $60 \pm 5 \%$. The rats were randomly divided into the sham operation group, the myocardial ischemia-reperfusion model group and the gastrodin group $(500 \mathrm{mg} / \mathrm{kg})$, with equal number of 20 rats per group. The rats in the gastrodin group were administered gastrodin twice daily 
by gavage for 7 days before model establishment and 3 days after model establishment. The rats in the sham operation and the myocardial ischemia-reperfusion model groups were given an equal volume of saline during the same time period. The rats used in this study were provided by the Experimental Animal Center of Nantong University with a permission number SCXX (Su) 2002-0019. All experimental procedures were performed in accordance with the Guide for the Care and Use of Laboratory Animals published by National Institutes of Health (Bethesda, MD, USA). The protocols were approved by the Animal Ethics Committee of the Affiliated Hospital of Nantong University (Nantong, China).

Establishment of myocardial ischemia model. To induce myocardial ischemia, the rats were anesthetized and subjected to open-chest surgery to expose the heart. Ligation of the left descending coronary artery was performed $2 \mathrm{~mm}$ below the border between the left atrium and the ventricle using 5/0 nylon suture. Myocardial reperfusion induced by ligature removal was performed in the myocardial ischemia-reperfusion model group. Those who only threaded but not ligated were the sham operation model group. Ischemia was confirmed by visually observed cyanosis and electrocardiogram demonstrating ST segment elevation and QRS widening. Successful establishment of the ischemia and reperfusion models was judged according to the criteria. Myocardial reperfusion induced by ligature removal was performed in the myocardial ischemiareperfusion model group. Those who only threaded but not ligated were the sham operation model group. All rats were euthanized on the 8th day after myocardial ischemia surgery, and the heart tissue was harvested.

Western blot analysis. Lysis of heart tissues was performed using the RIPA lysis buffer to extract proteins, followed by concentration measurement of the protein extracts and the BCA protein quantification kit was purchased from Thermo Fisher Scientific, Inc. (Waltham, MA, USA). A total of $5 \mu 1$ of protein was loaded per lane. The proteins in the sample were separated using SDS-PAGE gel electrophoresis and transferred to the membrane (NC membrane; MilliporeSigma, Burlington, MA, USA) using a wet system. The primary antibodies [monoclonal Bax antibody (1:1,500; cat. no. YS-28034R) and monoclonal Bcl-2 antibody (1:1,000; cat. no. YSm-10846M) purchased from Santa Cruz Biotechnology, Inc., Dallas, TX, USA, and monoclonal caspase-3 antibody (1:500; cat. no. ab131000) purchased from Abcam, Cambridge, UK] were added after the membrane was blocked with 5\% BSA, followed by incubation at $37^{\circ} \mathrm{C}$ overnight. Then, HRP-labeled goat antirabbit secondary antibody (1:1,000; cat. no. A0208, Beyotime Institute of Biotechnology, Shanghai, China) was added, and color was developed at $37^{\circ} \mathrm{C}$. The percentages of separation and stacking gels were 10 and $5 \%$, respectively.

Fluorescence-based reverse transcription-quantitative polymerase chain reaction $(R T-q P C R)$. Total RNA was extracted from the obtained cardiomyocytes in acccordance with the instructions of TRIzol kit (Invitrogen; Thermo Fisher Scientific, Inc.). The complementary DNA (cDNA) was synthesized via reverse transcription of the single stranded RNA in accordance with the instructions of cDNA kit (Toyobo
Co., Ltd., Osaka, Japan). SYBR-Green PCR kit (Toyobo Co., Ltd.) was used. Amplification of DNA was carried out, followed by analysis. The thermocycling conditions were: $42^{\circ} \mathrm{C} \times 15 \mathrm{~min}, 92^{\circ} \mathrm{C} \times 5 \mathrm{~min},\left(95^{\circ} \mathrm{C} \times 20 \mathrm{sec} \rightarrow 55^{\circ} \mathrm{C} \times 15 \mathrm{sec}\right.$ $\rightarrow 72^{\circ} \mathrm{C}$ x $\left.20 \mathrm{sec}\right) \times 30 \mathrm{sec}, 96^{\circ} \mathrm{C}$ x $30 \mathrm{sec}, 55^{\circ} \mathrm{C}$ x $30 \mathrm{sec}$, $95^{\circ} \mathrm{C}$ x $30 \mathrm{sec}$. All expression levels were calculated using the $2^{-\Delta \Delta C q}$ method (15). The primers used in RT-qPCR are shown in Table I.

Terminal deoxynucleotidyl transferase-mediated dUTP. As previously described (16), apoptosis was assessed using TUNEL assay (Roche Molecular Diagnostics, Pleasanton, CA, USA). Frozen sections (6 mm thickness) or cells were stained with DAPI (Thermo Fisher Scientific, Inc.) at room temperature for $5 \mathrm{~min}$, allowing for visualization of the nucleus. Monoclonal anti- $\alpha$-actinin antibody $(1: 1,000$; cat. no. sc-17829; Santa Cruz Biotechnology, Inc.) were added, followed by incubation at $4^{\circ} \mathrm{C}$ overnight. The sections were then incubated with goat anti-rat $\alpha$-actinin antibody at $37^{\circ} \mathrm{C}$ for $2 \mathrm{~h}$. Positive cells were counted using a fluorescence microscopy.

$H E$ staining. All specimens were fixed by formaldehyde, after which they were embedded in paraffin and routinely sectioned. The samples were then stained, followed by pathological examination under light microscope (Olympus Corp., Tokyo, Japan).

ELISA. Serum levels of IL-6, IL-10, TNF- $\alpha$ and IL-1 $\beta$ were measured using ELISA kits according to the instructions of the kits (Abcam).

Statistical analysis. GraphPad Prism Software (GraphPad Software, Inc., La Jolla, CA, USA) was used for statistical analysis. The count data were analyzed by $\chi^{2}$ test. One-way analysis of variance was used for comparison for multiple groups, and Fisher's Least Significant Difference test was the post-hoc test used after one-way analysis. All data were expressed as (mean \pm standard deviation). $\mathrm{P}<0.05$ was considered to indicate a statistically significant difference.

\section{Results}

Alleviation of myocardial ischemia-reperfusion injury by gastrodin. As shown in the TUNEL assay results (Table II and Fig. 1), the cardiomyocyte apoptotic rates in the sham operation, myocardial ischemia-reperfusion model and gastrodin groups were $2.1 \pm 0.8,24.2 \pm 8.5$, and $12.1 \pm 3.7 \%$, respectively. Compared with the other two groups, the atrial apoptosis rate of the gastrodin treatment group was higher than that of the sham operation group, but the myocardial apoptosis rate of the gastrodin treatment group was significantly lower than that of the myocardial ischemia-reperfusion model group $(\mathrm{P}<0.05)$.

Morphological changes of myocardial tissues were examined by HE staining, and the results are shown in Fig. 2. Cardiomyocytes in the sham operation group were neatly arranged. In the myocardial ischemia-reperfusion model group, the cardiomyocytes were in disordered arrangements. Widening gaps and inflammatory factor exudates were observed. The degree of cardiomyocyte damage 
Table I. Primers used in RT-qPCR assay.

\begin{tabular}{ll}
\hline Genes & \multicolumn{1}{c}{ Primer sequences } \\
\hline GAPDH & F: 5'-GACAACTITGGCATCGTGGA-3' \\
& R: 5'-ATGCAGGGATGATGTTCTGG-3 \\
Bax & F: 5'-AGACACCTGAGCTGACCTTGGAG-3' \\
& R: 5'-GTTGAAGTTGCCATCAGCAAACA-3' \\
Bcl-2 & F: 5'-TGAACCGGCATCTGCACAC-3' \\
& R: 5'-CGTCTTCAGAGACAGCCAGGAG-3' \\
Caspase-3 & F: 5'-CATACAGCGGAACTGTCGAT-3' \\
& R: 5'-GTTCAGCAAGGCGCATAGTG-3'
\end{tabular}

$\mathrm{F}$, forward; $\mathrm{R}$, reverse.

Table II. Cardiomyocyte apoptotic rate in the three groups (\%).

\section{Groups}

Sham operation $(n=20)$

Gastrodin (n=20)

Myocardial ischemia-reperfusion model $(\mathrm{n}=20) \quad 24.2 \pm 8.5^{\mathrm{a}, \mathrm{b}}$

${ }^{\mathrm{a}} \mathrm{P}<0.05$, compared with the sham operation group; ${ }^{\mathrm{b}} \mathrm{P}<0.05$, compared with the gastrodin group.
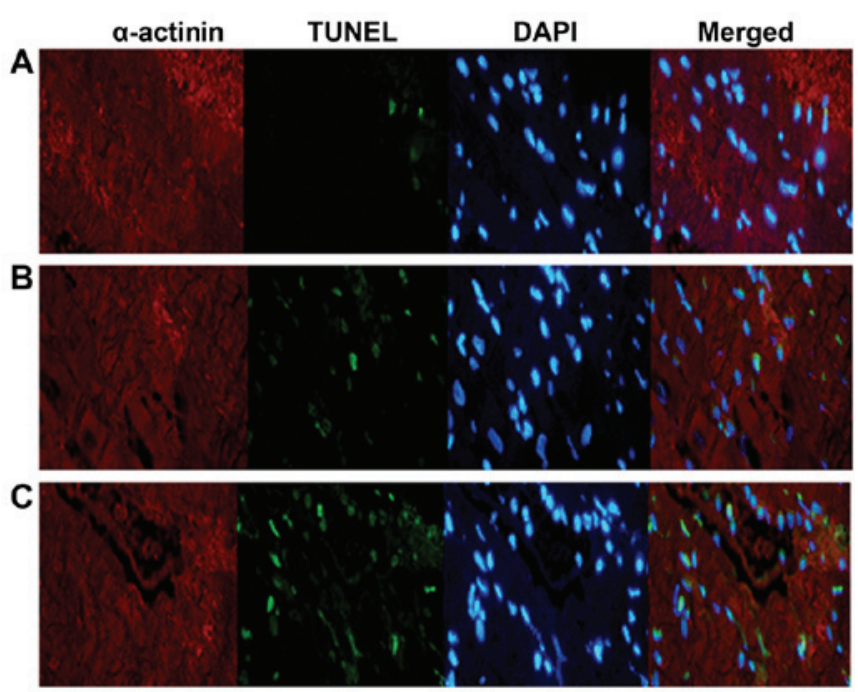

Figure 1. Cardiomyocyte apoptosis measured by TUNEL assay in (A) the sham operation, (B) the gastrodin, and (C) the myocardial ischemia-reperfusion model groups.

in the gastrodin group was significantly less than that in the myocardial ischemia-reperfusion model group.

Expression levels of proteins involved in cardiomyocyte pathways. As shown in the results of western blot analysis (Table III and Fig. 3), the expression levels of Bax and activated caspase- 3 were $0.38 \pm 0.16$ and $0.43 \pm 0.21$, respectively, in the gastrodingroup, as wellas $0.87 \pm 0.36$ and $0.93 \pm 0.36$ respectively,

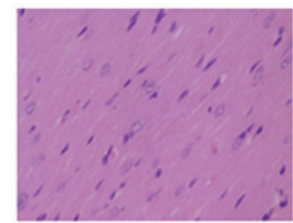

A

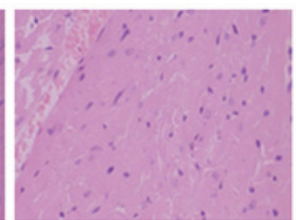

B

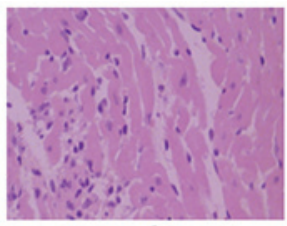

C
Figure 2. Morphology of cardiomyocytes examined by HE staining in (A) the sham operation, (B) gastrodin, and (C) myocardial ischemia-reperfusion model groups.

in the sham operation group as well as $1.42 \pm 0.28$ and $1.67 \pm 0.37$ respectively, in the myocardial ischemia-reperfusion model group. The differences between the two groups were statistically significant $(\mathrm{P}<0.05)$. The expressions of Bax and activated caspase- 3 protein in the gastrodin, sham operation and myocardial ischemia-reperfusion groups showed an increasing trend, and the differences between the groups were statistically significant $(\mathrm{P}<0.05)$. The expression level of $\mathrm{Bcl}-2$ in the gastrodin group increased to $0.59 \pm 0.24$.

mRNA expression levels of genes related to cardiomyocyte pathways. As shown in the RT-qPCR results (Table IV), the mRNA expression levels of pro-apoptotic genes Bax and activated caspase- 3 were $0.98 \pm 0.31$ and $0.99 \pm 0.40$, respectively, in the sham operation group, as well as $0.46 \pm 0.27$ and $0.55 \pm 0.19$, respectively, in the gastrodin group, and $1.24 \pm 0.45$ and $0.98 \pm 0.42$, respectively, in the cardiac ischemia-reperfusion group. The expression levels of Bax and activated caspase- 3 mRNA in the gastrodin, sham operation and myocardial ischemia-reperfusion groups showed an increasing trend, and the differences between the groups were statistically significant $(\mathrm{P}<0.05)$. The expression levels of the anti-apoptotic gene $\mathrm{Bcl}-2$ in the sham operation, gastrodin, and myocardial ischemia-reperfusion model groups were $0.97 \pm 0.38,0.89 \pm 0.36$ and $0.32 \pm 0.15$, respectively, $(\mathrm{P}<0.05)$.

Serum levels of inflammatory factors. As shown in the ELISA results (Table V), the serum levels of IL-6, TNF- $\alpha$ and IL-1 $\beta$ were $41 \pm 16,26 \pm 9$ and $37 \pm 14$, respectively, in the sham operation group, as well as $138 \pm 42,126 \pm 35$ and $145 \pm 42$, respectively, in the myocardial ischemia-reperfusion model group and $59 \pm 23,58 \pm 17$ and $49 \pm 15$ in the gastrodin group, respectively. The differences between the two groups were statistically significant $(\mathrm{P}<0.05)$. The serum levels of IL-10 were $88 \pm 17$ and $167 \pm 45$, respectively, in the myocardial ischemia-reperfusion model and gastrodin groups, and the difference was statistically significant. In the gastrodin group, the higher level of IL-10 was clearly opposite to the lower levels of IL-6, TNF- $\alpha$ and IL-1 $\beta$.

\section{Discussion}

Studies have shown that defects in many signaling pathways are associated with myocardial ischemia-reperfusion injury $(17,18)$. For example, the interaction between activated PI3K/AKT pathway and downstream target proteins, including Bcl-2 and Bax, promotes cell survival (19). If the PI3K/AKT pathway is dysregulated, alteration of cell signaling such as Bcl-2 can occur, eventually leading to apoptosis. Ke et al 
Table III. Expression levels of proteins involved in cardiomyocyte pathways (mean \pm standard deviation).

\begin{tabular}{lcccc}
\hline Groups & Bax & Bcl-2 & Bcl-2/Bax & Activated caspase-3 \\
\hline Sham operation $(\mathrm{n}=20)$ & $0.87 \pm 0.36$ & $0.92 \pm 0.25$ & $1.06 \pm 0.23$ & $0.93 \pm 0.36$ \\
Gastrodin $(\mathrm{n}=20)$ & $0.38 \pm 0.16^{\mathrm{a}}$ & $0.59 \pm 0.24^{\mathrm{a}}$ & $1.55 \pm 0.34$ & $0.43 \pm 0.21^{\mathrm{a}}$ \\
Myocardial ischemia-reperfusion model $(\mathrm{n}=20)$ & $1.42 \pm 0.28^{\mathrm{a}, \mathrm{b}}$ & $0.34 \pm 0.27^{\mathrm{a}, \mathrm{b}}$ & $0.31 \pm 0.14$ & $1.67 \pm 0.37^{\mathrm{a}, \mathrm{b}}$
\end{tabular}

${ }^{\mathrm{a}} \mathrm{P}<0.05$, compared with the sham operation group; ${ }^{\mathrm{b}} \mathrm{P}<0.05$, compared with the gastrodin group.

Table IV. mRNA expression levels of genes related to cardiomyocyte pathways (mean \pm standard deviation).

\begin{tabular}{lllc}
\hline Groups & Bax & Bcl-2 & Activated caspase-3 \\
\hline Sham operation $(\mathrm{n}=20)$ & $0.98 \pm 0.31$ & $0.97 \pm 0.38$ & $0.99 \pm 0.40$ \\
Gastrodin $(\mathrm{n}=20)$ & $0.46 \pm 0.27^{\mathrm{a}}$ & $0.89 \pm 0.36^{\mathrm{a}}$ & $0.55 \pm 0.19^{\mathrm{a}}$ \\
Myocardial ischemia-reperfusion model $(\mathrm{n}=20)$ & $1.24 \pm 0.45^{\mathrm{a}, \mathrm{b}}$ & $0.32 \pm 0.15^{\mathrm{a}, \mathrm{b}}$ & $0.98 \pm 0.42^{\mathrm{b}}$ \\
\hline
\end{tabular}

${ }^{\mathrm{a}} \mathrm{P}<0.05$, compared with the sham operation group; ${ }^{\mathrm{b}} \mathrm{P}<0.05$, compared with the gastrodin group.

Table V. Serum levels of inflammatory factors (mean \pm standard deviation).

\begin{tabular}{|c|c|c|c|c|}
\hline Groups & IL-6 (ng/l) & IL-10 (ng/l) & $\mathrm{TNF}-\alpha(\mathrm{ng} / \mathrm{l})$ & IL-1 $\beta(\mathrm{ng} / \mathrm{l})$ \\
\hline Sham operation $(n=20)$ & $41 \pm 16$ & $45 \pm 18$ & $26 \pm 9$ & $37 \pm 14$ \\
\hline Gastrodin $(n=20)$ & $59 \pm 23^{\mathrm{a}}$ & $167 \pm 45^{\mathrm{a}}$ & $58 \pm 17^{\mathrm{a}}$ & $49 \pm 15^{\mathrm{a}}$ \\
\hline Myocardial ischemia-reperfusion model $(n=20)$ & $138 \pm 42^{\mathrm{a}, \mathrm{b}}$ & $88 \pm 17^{\mathrm{a}, \mathrm{b}}$ & $126 \pm 35^{\mathrm{a}, \mathrm{b}}$ & $145 \pm 42^{\mathrm{a}, \mathrm{b}}$ \\
\hline
\end{tabular}

${ }^{\mathrm{a}} \mathrm{P}<0.05$, compared with the sham operation group; ${ }^{\mathrm{b}} \mathrm{P}<0.05$, compared with the gastrodin group.

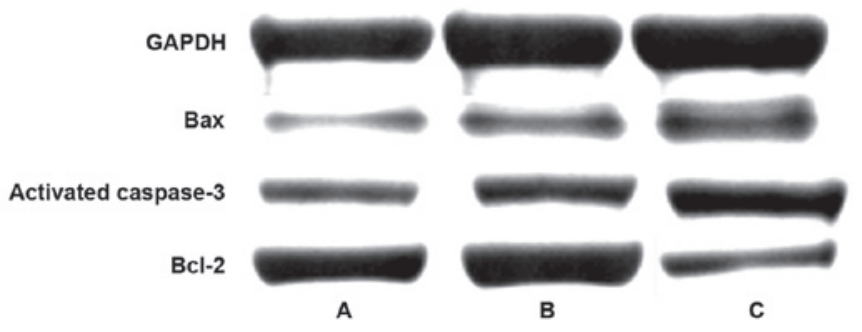

Figure 3. Western blot analysis assay of expression levels of proteins involved in cardiomyocyte pathways in (A) gastrodin, (B) sham operation, and (C) myocardial ischemia-reperfusion model groups.

reported that activation of the PI3K/AKT pathway protects against isoproterenol-induced myocardial ischemic injury (17). In another study, it was reported that physiological heart growth was induced by activating the PI3K/AKT pathway (20).

Some studies suggested that myocardial ischemia-reperfusion may aggravate cardiac dysfunction and myocardial cell damage, leading to inflammation and partial apoptosis. It is important to explore the underlying mechanism in order to prevent myocardial ischemia-reperfusion injury. According to literature, multiple factors such as calcium overload, neutrophil aggregation and cardiomyocyte apoptosis are involved in myocardial ischemia-reperfusion injury $(21,22)$.
Gastrodin is an effective monomer composition extracted from rhizome gastrodiae, which demonstrates various pharmacological effects. It not only improves microcirculation and general circulatory functions but also possesses neuroprotective effects and suppresses inflammatory factors. Myocardial ischemia is a serious condition that normally leads to devastating consequences such as necrosis and apoptosis (23). In this study, myocardial ischemia-reperfusion injury was investigated, aimed at providing more treatment information for this disease. Results from this study showed that the cardiomyocyte apoptotic rate in the myocardial ischemia-reperfusion model group increased substantially, compared with that in the sham operation group. Gastrodin significantly reduced cardiomyocyte apoptosis as suggested by the lower apoptotic rate in the gastrodin group, compared with the myocardial ischemia-reperfusion model group.

In this study, the effect of gastrodin on expression of activated caspase-3, Bax and Bcl-2 was also explored. Caspase-3 is capable of digesting specific protein substrates, thereby inhibiting DNA repair and leading to chromosomal DNA fragmentation and cell death. Members of the Bcl-2 family proteins play a critical role in regulating the autonomous apoptotic pathway. It is known that Bax and Bcl-2 can migrate to different parts of the cell to exert a role of signal transduction, thereby regulating cell survival or apoptosis (24). The results 
showed that gastrodin significantly downregulated expression of Bax and activated caspase- 3 but upregulated Bcl-2 expression in the gastrodin group, compared with the myocardial ischemia-reperfusion model group. These findings suggested that gastrodin has significant protective effects in myocardial ischemia-reperfusion injury.

Recent studies demonstrated that inflammatory lesions also played an important role in myocardial ischemia-reperfusion injury $(25,26)$. IL-6 is a cytokine that is mainly secreted from monocytes, macrophages and T/B lymphocytes. It can induce the expression of $I C A M-1$ gene in cardiomyocytes and promote the entry of granulocytes into the ischemic region. The overexpression of ICAM-1 mRNA participates in LFA-1 signaling transduction on the surface of neutrophil leading to adhesion of neutrophils and endothelial cells. It also mediates neutrophil infiltration and exacerbates inflammatory responses by increasing pro-inflammatory factors $(27,28)$. Pro-inflammatory cytokines TNF- $\alpha$ and IL- $1 \beta$ also play an important role in apoptotic processes. To assess anti-inflammatory effects of gastrodin, the expression levels of IL-6, IL-10, TNF- $\alpha$ and IL-1 $\beta$ were measured. The results showed that there were statistically significant differences in the expression of TNF- $\alpha$, IL-6, IL-10 and IL-1 $\beta$ between the groups. IL-1 $\beta$, TNF- $\alpha$ and IL- 6 were all upregulated in the myocardial ischemia-reperfusion model group, suggesting that myocardial ischemia-reperfusion may increase myocardial injury through inflammatory responses. Compared with the myocardial ischemia-reperfusion model group, gastrodin significantly reduced the expression of these cytokines in the gastrodin group. IL-10, as a multifunctional negative regulator, is involved in regulation of a variety of biological processes and plays a protective role. In this study, the expression level of IL-10 increased substantially in the gastrodin group, compared with the myocardial ischemia-reperfusion model group. It may play a role in protecting ischemic cardiomyocytes from apoptosis by suppressing inflammation. Findings in this study suggested that gastrodin may reduce inflammatory damage by suppressing inflammatory factors, thereby protecting the myocardial tissue.

In this study, it was revealed that gastrodin can decrease the release of inflammatory factors while increase the expression of protective cytokines. It reduced cardiomyocyte apoptosis by inhibiting apoptotic signaling, thereby alleviating myocardial ischemia-reperfusion injury. Therefore, findings in this study suggested that gastrodin can provide protection against myocardial ischemia-reperfusion injury, which may serve as a reference for better treatment of myocardial ischemia-reperfusion injury.

\section{Acknowledgements}

Not applicable.

\section{Funding}

No funding was received.

\section{Availability of data and materials}

The datasets used and/or analyzed during the current study are available from the corresponding author on reasonable request.

\section{Authors' contributions}

XH drafted the manuscript. XH, HS and KL conceived and designed the study. LZ, FW and QY performed PCR. KL and LZ were responsible for animal model construction. All authors read and approved the final manuscript.

\section{Ethics approval and consent to participate}

This study was approved by Affiliated Hospital of Nantong University (Nantong, China).

\section{Patient consent for publication}

Not applicable.

\section{Competing interests}

The authors declare that they have no competing interests.

\section{References}

1. Steg PG, Greenlaw N, Tendera M, Tardif JC, Ferrari R, Al Zaibag M, Dorian P, Hu D, Shalnova S, Sokn FJ, et al; Prospective Observational Longitudinal Registry of Patients with Stable Coronary Artery Disease (CLARIFY) Investigators: Prevalence of anginal symptoms and myocardial ischemia and their effect on clinical outcomes in outpatients with stable coronary artery disease: data from the international observational CLARIFY registry. JAMA Intern Med 174: 1651-1659, 2014.

2. Cheng D, Zhu C, Cao J and Jiang W: The protective effects of polyphenols from jujube peel (Ziziphus Jujube Mill) on isoproterenol-induced myocardial ischemia and aluminum-induced oxidative damage in rats. Food Chem Toxicol 50: 1302-1308, 2012.

3. Misumida N, Kobayashi A, Saeed M, Fox JT and Kanei Y: Electrocardiographic left ventricular hypertrophy as a predictor for nonsignificant coronary artery disease in patients with non ST segment elevation myocardial infarction. Angiology 67: 27-33, 2016

4. Sung HK, Chan YK, Han M, Jahng JWS, Song E, Danielson E, Berger T, Mak TW and Sweeney G: Lipocalin-2 (NGAL) attenuates autophagy to exacerbate cardiac apoptosis induced by myocardial ischemia. J Cell Physiol 232: 2125-2134, 2017.

5. Panza JA, Holly TA, Asch FM, She L, Pellikka PA, Velazquez EJ, Lee KL, Borges-Neto S, Farsky PS, Jones RH, et al: Inducible myocardial ischemia and outcomes in patients with coronary artery disease and left ventricular dysfunction. J Am Coll Cardiol 61: 1860-1870, 2013.

6. Patel RD and Saver JL: Evolution of reperfusion therapies for acute brain and acute myocardial ischemia: a systematic, comparative analysis. Stroke 44: 94-98, 2013.

7. Zhang J, Yu XH, Yan YG, Wang C and Wang WJ: PI3K/Akt signaling in osteosarcoma. Clin Chim Acta 444: 182-192, 2015.

8. Kim HJ, Moon KD, Lee DS and Lee SH: Ethyl ether fraction of Gastrodia elata Blume protects amyloid beta peptide-induced cell death. J Ethnopharmacol 84: 95-98, 2003.

9. Kim HJ, Moon KD, Oh SY, Kim SP and Lee SR: Ether fraction of methanol extracts of Gastrodia elata, a traditional medicinal herb, protects against kainic acid-induced neuronal damage in the mouse hip-pocampus. Neurosci Lett 314: 65-68, 2001.

10. Park S, da Kim S and Kang S: Gastrodia elata Blume water extracts improve insulin resistance by decreasing body fat in dietinduced obese rats: vanillin and 4-hydroxybenzaldehyde are the bioactive candidates. Eur J Nutr 50: 107-118, 2011.

11. Xu X, Lu Y and Bie X: Protective effects of gastrodin on hypoxia-induced toxicity in primary cultures of rat cortical neurons. Planta Med 73: 650-654, 2007.

12. Dai JN, Zong Y, Zhong LM, Li YM, Zhang W, Bian LG, Ai QL, Liu YD, Sun J and Lu D: Gastrodin inhibits expression of inducible NO synthase, cyclooxygenase-2 and proinflammatory cytokines in cultured LPS-stimulated microglia via MAPK pathways. PLoS One 6: e21891, 2011. 
13. Shu C, Chen C, Zhang DP, Guo H, Zhou H, Zong J, Bian Z, Dong X, Dai J, Zhang Y and Tang Q: Gastrodin protects against cardiac hypertrophy and fibrosis. Mol Cell Biochem 359: 9-16, 2012.

14. Zhao X, Zou Y, Xu H, Fan L, Guo H, Li X, Li G, Zhang X and Dong M: Gastrodin protect primary cultured rat hippocampal neurons against amyloid-beta peptide-induced neurotoxicity via ERK1/2-Nrf2 pathway. Brain Res 1482: 13-21, 2012.

15. Livak KJ and Schmittgen TD: Analysis of relative gene expression data using real time quantitative PCR and the 2(-Delta Delta C(T)) method. Methods 25: 402-408, 2001.

16. Abbate A, Salloum FN, Van Tassell BW, Vecile E, Toldo S, Seropian I, Mezzaroma E and Dobrina A: Alterations in the interleukin-1/interleukin-1 receptor antagonist balance modulate cardiac remodeling following myocardial infarction in the mouse. PLoS One 6: e27923, 2011.

17. Ke Z, Wang G, Yang L, Qiu H, Wu H, Du M, Chen J, Song J, Jia X and Feng L: Crude terpene glycoside component from Radix paeoniae rubra protects against isoproterenol-induced myocardial ischemic injury via activation of the PI3K/AKT/mTOR signaling pathway. J Ethnopharmacol 206: 160-169, 2017.

18. Cui G, Shan L, Hung M, Lei S, Choi I, Zhang Z, Yu P, Hoi P, Wang Y and Lee SM: A novel Danshensu derivative confers cardioprotection via PI3K/Akt and Nrf2 pathways. Int J Cardiol 168: 1349-1359, 2013.

19. Ouyang ZH, Wang WJ, Yan YG, Wang B and Lv GH: The PI3K/Akt pathway: A critical player in intervertebral disc degeneration. Oncotarget 8: 57870-57881, 2017.

20. De Los Santos S, García-Pérez V, Hernández-Reséndiz S, Palma-Flores C, González-Gutiérrez CJ, Zazueta C, Canto P and Coral-Vázquez RM: (-)-Epicatechin induces physiological cardiac growth by activation of the PI3K/Akt pathway in mice. Mol Nutr Food Res 61: 61, 2017.

21. Correa F, Buelna-Chontal M, Chagoya V, García-Rivas G, Vigueras RM, Pedraza-Chaverri J, García-Niño WR, Hernández-Pando R, León-Contreras JC and Zazueta C: Inhibition of the nitric oxide/cyclic guanosine monophosphate pathway limited the cardioprotective effect of post-conditioning in hearts with apical myocardial infarction. Eur J Pharmacol 765 : 472-481, 2015
22. Kambara T, Shibata R, Ohashi K, Matsuo K, Hiramatsu-Ito M, Enomoto T, Yuasa D, Ito M, Hayakawa S, Ogawa $\mathrm{H}$, et al: $\mathrm{C} 1 \mathrm{q} /$ tumor necrosis factor-related protein 9 protects against acute myocardial injury through an adiponectin receptor I-AMPK-dependent mechanism. Mol Cell Biol 35: 2173-2185, 2015.

23. Broughton BR, Reutens DC and Sobey CG: Apoptotic mechanisms after cerebral ischemia. Stroke 40: e331-e339, 2009.

24. Pape M,Engelhard K, Eberspächer E, Hollweck R, Kellermann K, Zintner S, Hutzler P and Werner C: The long-term effect of sevoflurane on neuronal cell damage and expression of apoptotic factors after cerebral ischemia and reperfusion in rats. Anesth Analg 103: 173-179, 2006.

25. Smith IC, Vigna C, Levy AS, Denniss SG, Rush JW and Tupling AR: The effects of buthionine sulfoximine treatment on diaphragm contractility and SERCA pump function in adult and middle aged rats. Physiol Rep 3: e12547, 2015.

26. Komin N, Moein M, Ellisman MH and Skupin A: Multiscale modeling indicates that temperature dependent $\left[\mathrm{Ca}^{2+}\right]$ i spiking in astrocytes is quantitatively consistent with modulated SERCA activity. Neural Plast 2015: 683490, 2015.

27. Yan J, Schmid E, Hosseinzadeh Z, Honisch S, Shumilina E, Fuchs J and Lang F: Impact of Janus kinase 3 on cellular Ca release, store operated $\mathrm{Ca}(2+)$ entry and $\mathrm{Na}(+) / \mathrm{Ca}(2+)$ exchanger activity in dendritic cells. Cell Physiol Biochem 36: 2287-2298, 2015.

28. Wiernicki B, Rozwadowska N, Malcher A, Kolanowski T, Zimna A, Rugowska A and Kurpisz M: Human myoblast transplantation in mice infarcted heart alters the expression profile of cardiac genes associated with left ventricle remodeling. Int J Cardiol 202: 710-721, 2016.

(1) (9) This work is licensed under a Creative Commons EY NO NO Attribution-NonCommercial-NoDerivatives 4.0 International (CC BY-NC-ND 4.0) License. 